\title{
JUVENTUD Y CONFIANZA SOCIAL EN CHILE
}

\author{
JORGE BAEZA CORREA*
}

\begin{abstract}
RESUMEN
La finalidad de este artículo es un intento de sistematizar y abrir desde el conocimiento acumulado algunas reflexiones sobre la juventud chilena y la confianza social. Este trabajo se concentra en los temas de la confianza generalizada y la confianza en las instituciones, dos aspectos fundamentales de la construcción democrática de un país, dado que constituyen datos significativos sobre la cohesión social. Los datos sobre confianza muestran una juventud no diferente a la tendencia general de la población chilena. Los jóvenes no se ubican en una posición extrema de total desconfianza con el otro desconocido, pero sí de clara conducta a la defensiva. Es claro, además, que es estable a través del tiempo una bajísima confianza en las instituciones vinculadas a instancias políticas y judiciales, pero se confía en las instituciones educacionales. En este marco de desconfianza, se aprecia una juventud, que transita desde una crítica sistémica hacia una validación del emprendimiento, lo que lleva a una cohesión social ya no construida sobre la homogeneidad, sino sobre la base de que a nadie se le discrimina y donde el esfuerzo individual no descarta el apoyo público, sino que al contrario, lo demanda como una compensación al trabajo individual.
\end{abstract}

PALABRAS CLAVE: CONFIANZA, JUVENTUD, COHESIÓN SOCIAL

* Sociólogo y Doctor en Ciencias de la Educación. Académico e investigador del Centro de Estudios en Juventud (CEJU) de la Universidad Católica Silva Henríquez, Chile. Correo electrónico:jbaeza@ucsh.cl.

El presente artículo es parte del trabajo de revisión documental del proyecto de investigación Fondecyt Nº1100649: «Vinculaciones entre la construcción y deconstrucción de la confianza y la cohesión social en jóvenes estudiantes de educación secundaria: lineamientos para fortalecer la democracia», donde el autor es el investigador responsable. 


\title{
JUVENTUDE E CONFIANÇA SOCIAL NO CHILE
}

\section{RESUMO}

O objetivo deste artigo é sistematizar e propor, a partir do conhecimento acumulado, algumas reflexões sobre a juventude chilena e a confiança social. Este trabalho se concentra nos temas da confiança em geral e da confiança nas instituições, dois aspectos fundamentais da construção democrática de um país, uma vez que constituem dados significativos sobre a coesão social. Os dados sobre confiança mostram uma juventude não diferente da tendência geral da população chilena. Os jovens não se colocam numa posição extrema de total desconfiança com o outro desconhecido, mas sim de clara conduta defensiva. Fica evidente, também, a persistência ao longo do tempo de uma baixa confiança nas instituições vinculadas a instâncias políticas e judiciais, porém, evidencia-se uma confiança nas instituições educacionais. Neste marco de desconfiança, observa-se uma juventude que transita desde a crítica sistêmica até a validação do empreendimento, o que acarreta a uma coesão social construída não sobre a homogeneidade, mas sobre o pressuposto de que ninguém é discriminado, onde o esforço individual não descarta o apoio público, mas ao contrário, exige-o como compensação ao trabalho individual.

PALAVRAS CHAVE: CONFIANÇA, JUVENTUDE, COESÃO SOCIAL

\section{YOUTH AND SOCIAL CONFIDENCE IN CHILE}

\begin{abstract}
The purpose of this article is an attempt to systematize and open from the accumulated knowledge of some reflections on Chilean youth and the social confidence. This article focuses on the subjects of generalized confidence and the confidence in institutions, two fundamental aspects of the democratic construction of a country, since they constitute significant data on social cohesion. The data on confidence shows that youth are non-different from the general tendency of the Chilean population. Youth, are not located in an extreme position of total distrust with strangers, but of a clear defensive conduct. In addition, it is clear that a very low confidence in the tie institutions to political and judicial instances is stable through time, but one trusts the educational institutions. In this frame of distrust, a youth is appraised, that journeys from a systemic critic towards a validation of the beginning, which entails a social cohesion no longer constructed on the homogeneity, but on the base on which anybody is discriminated and where the individual effort does not discard the public support, but the opposite, it demands a compensation to the individual work.
\end{abstract}

KEY WORDS: CONFIDENCE, YOUTH, SOCIAL COHESION 


\section{INTRODUCCIÓN}

LA CONFIANZA CONSTITUYE HOY en día un tema con bastante presencia en los trabajos de distintas disciplinas de las ciencias sociales, no obstante ello, aún sigue siendo un tema que requiere de mayor desarrollo y reflexión dada su relevancia. «La confianza —indica el texto del Latinobarómetro 2010 (2010:70) — es uno de los indicadores más críticos del análisis de la democracia. Es un tema que no está sujeto a políticas públicas, pertenece al ámbito de la cultura de los pueblos, está forjado por la historia y las experiencias, y parece ser el punto de tope de muchas transformaciones [...] parece ser un talón de Aquiles del desarrollo».

La importancia de la confianza radica en su fuerte vinculación con la cohesión social, ambas son conceptos íntimamente asociados, y a su vez, pilares fundamentales de una sociedad democrática moderna. La participación de ciudadanos en los asuntos públicos, en la vida de la comunidad y el desarrollo de la sociedad, requieren de una confianza entre los habitantes de una nación que se exprese en redes, en tejido social, que dé cuenta de una cohesión entre sus miembros.

Chile presenta una verdadera erosión de las instituciones de protección social —el Estado cada vez más se desprende de sus responsabilidades en educación, salud, vivienda y previsión-, lo que ha significado una profunda transformación cultural en la sociedad. El entramado social se ha vuelto más frágil y el concepto de comunidad se ha resquebrajado. La vida personal se ve caracterizada por el despliegue de la individualización, donde cada vez más las personas deben definir por sí mismas sus objetivos, valores y proyectos, lo que produce agobio y retracción social en las personas. Los cambios culturales en este sentido han creado oportunidades pero también dificultades para la convivencia cotidiana; se han diversificado los modos de vida, pero en muchos casos también se trata de una diversidad disociada, que en definitiva hace que Chile enfrente el desafío cultural de fortalecer las capacidades para reconocerse en un nosotros común.

\section{CONFIANZA SOCIAL: \\ LA CONFIANZA GENERALIZADA EN LOS OTROS}

La confianza es un tema de larga data en las ciencias sociales; Georg Simmel afirmaba en 1906 que «sin la confianza general que los sujetos tienen entre ellos, la sociedad se desintegraría» (Simmel, 1906:178). 
Para este autor muy pocas relaciones sociales se basan en el conocimiento de la otra persona, por ello, si la confianza no fuese tan fuerte, muy pocas relaciones durarían. En este sentido, es importante distinguir entre confianza particularizada y confianza generalizada. Siguiendo a Eric Uslaner (2002), la confianza 'particularizada' es cuando nos fiamos de personas que conocemos o son similares a nosotros, y 'generalizada', si creemos que podemos confiar en la mayoría de la gente.

La confianza social, o confianza generalizada, se define como confianza en desconocidos acerca de los que se carece de información. La confianza generalizada «es un tipo de confianza muy sensible a los comportamientos oportunistas. La confianza social o confianza generalizada es confianza en extraños, en desconocidos acerca de los que se carece de información respecto de si son o no dignos de confianza» (Herreros, 2004:607).

Respecto a la confianza social es posible distinguir en la literatura dos tipos de aproximaciones. Una de un carácter más bien relacional y una segunda más centrada en aspectos culturales. En la primera, la confianza es una decisión racional basada en un cálculo estratégico, una predicción sobre la conducta de los otros. La confianza es, por lo tanto, una decisión que supone un riesgo, ya que la persona que confía no tiene la seguridad en el otro. La segunda aproximación adopta una perspectiva culturalista sobre la confianza social, que explica la decisión de confiar en las normas y valores compartidos por los individuos en un determinado contexto. La confianza sería más una conducta favorable hacia la sociedad que una predicción del comportamiento de los otros.

En esta segunda perspectiva se ubica el trabajo de Robert Putnam, para quien la confianza estaría basada en las normas de reciprocidad y redes de compromiso cívico, entendidas en los siguientes términos: yo hago esto ahora por ti, sin esperar nada a cambio de inmediato y quizá sin conocerte, pero confiando en que más adelante tú o algún otro me devuelva el favor.

La confianza interpersonal, por lo tanto, no es la confianza en las personas con las que mantenemos vínculos estrechos, como los familiares y los amigos, sino con la gente en general. La reciente publicación del Latin American Public Opinion Project (LAPOP, 2010) para medir los niveles de confianza interpersonal en las Américas lo hace a partir de una sola pregunta, con el siguiente enunciado y alternativas: «Ahora, hablando de la gente de por aquí, ¿diría que la gente de su comunidad es?: (1) muy confiable (2) algo confiable (3) poco confia- 
ble (4) nada confiable». Los resultados a esta consulta se distribuyen de la siguiente manera:

\begin{tabular}{|c|c|}
\hline Ahora, hablando de la gente de por aquí, ¿diría que la gente de su comunidad es: \\
\hline Muy confiable & $22,0 \%$ \\
\hline Algo confiable & $42,3 \%$ \\
\hline Poco confiable & $25,4 \%$ \\
\hline Nada confiable & $10,3 \%$ \\
\hline
\end{tabular}

Fuente: LAPOP, 2010.

Estos datos reafirman una vez más que en nuestra región prima una baja confianza en los otros. Estos promedios, si se examinan las tendencias en los niveles de confianza interpersonal de las últimas cuatro rondas de encuestas en los once países para los que se tienen datos desde 2004, se han mantenido muy estables a lo largo del tiempo (59.1\% en 2004; 58.8\% en 2006; 58.8\% en 2008 y 59.6\% en 2010), el promedio total del nivel de confianza interpersonal en las Américas no ha experimentado ningún cambio estadísticamente significativo desde 2004, lo que a juicio de los autores del estudio es lo que podría esperarse para una variable fundamental de cultura.

Los resultados del Latinobarómetro 2010, son absolutamente coincidente con los anteriores. El indicador de confianza interpersonal en los datos del Latinobarómetro prácticamente no tiene variaciones significativas a lo largo de los años. Este fluctúa alrededor de los veinte puntos porcentuales.

Hablando en general, ¿diría Ud. que se puede confiar en la mayoría de las personas o que uno nunca es lo suficientemente cuidadoso en el trato con los demás? Aquí sólo «se puede confiar en la mayoría de las personas».

\begin{tabular}{|c|c|c|c|c|c|c|c|c|c|c|c|}
\hline 1996 & 2000 & 2001 & 2002 & 2003 & 2004 & 2005 & 2006 & 2007 & 2008 & 2009 & 2010 \\
\hline 20 & 16 & 17 & 19 & 17 & 16 & 19 & 22 & 17 & 21 & 21 & 20 \\
\hline
\end{tabular}

Fuente: Latinobarómetro, 2010.

Los datos para Chile en esta materia lo ubican por debajo del promedio general; para el 2010 en el Latinobarómetro es sólo un 17\% el que sostiene que «se puede confiar en la mayoría de las personas». Países como República Dominicana y Uruguay casi duplican a Chile, con un $31 \%$ y $30 \%$, respectivamente.

El estudio realizado por FLACSO/IPSOS (2010), en el marco del proyecto Gobernabilidad y Convivencia Democrática en América Latina, agrega a los datos anteriores que en América Latina, pese a que existe 
una tendencia a considerar que las personas que viven en su comunidad tienen valores similares a los propios (66\%) y que si se tiene un problema habrá alguien dispuesto a ayudar (68\%), los entrevistados tienden a manifestar desconfianza casi con tanta frecuencia (47\%) como a no tenerla (53\%) con respecto a quienes habitan en su comunidad.

En el caso de Chile, el 57\% de las respuestas considera que las personas de la comunidad donde se vive son mayoritariamente honestas, pero de ese mismo grupo de personas hay un $36,6 \%$ que considera que «casi nunca» o «nunca» podría en general confiar en la gente de su comunidad:

\begin{tabular}{|c|c|c|c|c|}
\hline \multirow{2}{*}{$\begin{array}{l}\text { Respuesta de Chile a la pregun- } \\
\text { ta: «¿Usted considera que las } \\
\text { personas de su comunidad son } \\
\text { mayoritariamente honestas?» }\end{array}$} & Sí & No & \multicolumn{2}{|c|}{ Igual } \\
\hline & 57,8 & 39,2 & \multicolumn{2}{|c|}{3,0} \\
\hline \multirow{2}{*}{$\begin{array}{l}\text { Respuesta de Chile a la pregun- } \\
\text { ta «¿Considera usted que en su } \\
\text { comunidad...»? En general, se } \\
\text { puede confiar en la gente }\end{array}$} & $\begin{array}{c}\text { Casi } \\
\text { nunca }\end{array}$ & Nunca & $\begin{array}{c}\text { Casi } \\
\text { Siempre } \\
\end{array}$ & Siempre \\
\hline & 7,0 & 29,6 & 54,0 & 9,4 \\
\hline
\end{tabular}

Fuente: FLACSO/IPSOS, 2010.

Estudios de este mismo orden dentro de Chile confirman lo ya expresado. La encuesta CERC (Hunneus 2010), indica con estabilidad desde 1988 que la respuesta «se puede confiar en la mayoría de la gente» se ubica alrededor del $20 \%$, mientras que «nunca se es suficientemente cuidadoso» se encuentra siempre cercano al $80 \%$.

El estudio de CIEPLAN/IFHC (2007) sobre Chile en la Encuesta de Cohesión Social en América Latina (EcosociaAL, 2007), también confirma las tendencias anteriores. Sólo un 10\% de los chilenos sostiene que «se puede confiar en la mayoría de las personas». Este estudio agrega a ello cuatro importantes datos.

\begin{tabular}{|c|c|c|c|c|c|c|c|}
\hline \multicolumn{6}{|c|}{ Porcentaje que dice: «Se puede confiar en la mayoría de las personas» en Chile. } \\
\hline \multicolumn{2}{|c|}{ Localidad } & \multicolumn{2}{|c|}{ Sexo } & \multicolumn{5}{c|}{ Educación } \\
\hline Regiones & Santiago & Mujer & Hombre & Básica & Secun I & Secun II & Superior \\
\hline 11 & 9 & 8 & 13 & 3 & 4 & 8 & 19 \\
\hline
\end{tabular}

Fuente: CIEPLAN/IFHC, 2007.

\begin{tabular}{|c|c|c|c|c|}
\hline \multicolumn{5}{|c|}{ Porcentaje que dice: «Se puede confiar en la mayoría de las personas» en Chile. } \\
\hline $18-24$ años & $25-34$ años & $35-44$ años & $45-54$ años & 55 o más \\
\hline 15 & 9 & 9 & 11 & 8 \\
\hline
\end{tabular}

Fuente: CIEPLAN/IFHC, 2007. 
Aquí también se entregan datos más diferenciados que permiten ir acercándose a la situación de los jóvenes en Chile y la confianza social.

\begin{tabular}{|c|c|c|c|c|c|c|c|}
\hline \multicolumn{9}{|c|}{ Porcentaje que dice: «La gente actúa correctamente con uno» en Chile. } \\
\hline \multicolumn{2}{|c|}{ Localidad } & \multicolumn{2}{|c|}{ Sexo } & \multicolumn{4}{c|}{ Educación } \\
\hline Regiones & Santiago & Mujer & Hombre & Básica & Secun I & Secun II & Superior \\
\hline 38 & 30 & 33 & 35 & 19 & 21 & 29 & 52 \\
\hline
\end{tabular}

Fuente: CIEPLAN/IFHC, 2007.

\begin{tabular}{|c|c|c|c|c|}
\hline \multicolumn{5}{|c|}{ Porcentaje que dice: «La gente actúa correctamente con uno» en Chile. } \\
\hline 18-24 años & $25-34$ años & $35-44$ años & $45-54$ años & 55 o más \\
\hline 43 & 33 & 35 & 32 & 29 \\
\hline
\end{tabular}

Fuente: CIEPLAN/IFHC, 2007.

Este conjunto de datos, está indicando - aunque no es muy importante la brecha- que la desconfianza es mayor en la ciudad capital que en regiones, que los hombres son más confiados que las mujeres y que las personas de mayor educación poseen mayor confianza en los otros. Se agrega, además, que a menor edad es también menor la desconfianza.

En Chile, los datos sobre juventud y confianza social o interpersonal no son muy abundantes. La V Encuesta Nacional de Juventud (INJUV, 2006) incluyó una pregunta al respecto, pero ella no tuvo continuidad en la VI Encuesta realizada el 2009. Ni tampoco tiene datos anteriores. La alta y total confianza es en uno de cada cuatro jóvenes.

\begin{tabular}{|l|c|c|c|}
\hline $\begin{array}{l}\text { Señala tu grado } \\
\text { de confianza: }\end{array}$ & $\begin{array}{c}\text { Confianza total }+ \\
\text { bastante confianza }\end{array}$ & Algo de confianza & $\begin{array}{c}\text { Poca confianza }+ \\
\text { nada de confianza }\end{array}$ \\
\hline $\begin{array}{l}\text { En las personas } \\
\text { en general }\end{array}$ & 22,5 & 55,1 & 19,8 \\
\hline
\end{tabular}

Fuente: V Encuesta Nacional de Juventud, Chile, 2006.

Los Estudios de Valores en Jóvenes Estudiantes de Educación Secundaria en la Región Metropolitana de Chile, de Baeza y Sandoval (2007 y 2009) incluyen también una consulta sobre el particular.

\begin{tabular}{|l|c|c|}
\hline \multirow{2}{*}{ En general tú dirías: } & \multicolumn{2}{|c|}{ Estudios } \\
\cline { 2 - 3 } & $\mathbf{2 0 0 7}$ & $\mathbf{2 0 0 9}$ \\
\hline Que se puede confiar en la mayoría de la gente & $7,7 \%$ & $4,1 \%$ \\
\hline $\begin{array}{l}\text { Que nunca se sabe las intenciones de los otros } \\
\text { (confiar pero con límites) }\end{array}$ & $77,9 \%$ & $84,2 \%$ \\
\hline Que es mejor no confiar en nadie & $14,4 \%$ & $11,8 \%$ \\
\hline
\end{tabular}

Fuente: Baeza y Sandoval, 2007 y 2009. 
En los resultados del estudio más reciente de Baeza y Sandoval, y en coincidencia con el anterior, las mujeres en mayor porcentaje que los hombres consideran que «es mejor no confiar en nadie»: 9,6\% contra $14 \%$; mientras en los otros aspectos como la edad y el estrato social, presentan medidas muy similares en su interior.

Una información más indirecta sobre el tema es la que se puede encontrar en la última Encuesta Nacional de Juventud en Chile (2009). En este caso, no hablando de la confianza en los otros en general, sino como ven los jóvenes la confianza de los adultos sobre la juventud. Para un $10,8 \%$ de los jóvenes «la falta de confianza que tienen los adultos en las y los jóvenes» es la sexta prioridad de los problemas que afectan a la juventud. En otras palabras, los jóvenes no sólo demuestran desconfianza, sino que sienten también que se desconfía de ellos. Situación que es más notoria en los hombres (9,0\% versus $12,7 \%$ en las mujeres) y en los de menor edad (13,8 en el grupo de 15 a 19 años versus 8,3\% en el grupo de 25 a 29 años). En cuanto a estrato social es bastante similar, pero con una leve tendencia a aumentar al bajar en estrato.

Este dato, ahora consultado por «los problemas propios más importantes», sube a un $15,5 \%$. La falta de confianza que tienen los adultos en las y los jóvenes constituye la quinta prioridad, por ejemplo, por sobre los problemas de calidad de la educación:

\begin{tabular}{|c|c|c|c|c|c|}
\hline \multirow{4}{*}{$\begin{array}{c}\text { TOTAL } \\
15,5\end{array}$} & \multicolumn{2}{|c|}{ Sexo } & \multicolumn{3}{c|}{ Tramo Etario } \\
\cline { 2 - 6 } & Hombre & Mujer & $15-19$ & $20-24$ & $25-29$ \\
\cline { 2 - 6 } & 16,9 & 14,1 & 23,8 & 12,5 & 9,4 \\
\cline { 2 - 6 } & \multicolumn{6}{|c|}{ Nivel Socioeconómico } \\
\cline { 2 - 6 } & ABC1 & C2 & C3 & D & E \\
\cline { 2 - 6 } & 23,8 & 15,8 & 12,6 & 15,3 & 15,8 \\
\hline
\end{tabular}

Fuente: VI Encuesta Nacional de Juventud, Chile, 2009

Estos últimos datos son bastante coincidentes con los estudios de LAPOP (2010), donde mediante un modelo de regresión lineal se calcula los determinantes principales de la confianza interpersonal. Los resultados muestran que las personas mayores, aquéllos que viven en pequeñas ciudades, aquéllos que son más ricos y aquéllos con mayor educación tienen mayores niveles de confianza interpersonal. Por el contrario, las mujeres tienen un nivel menor de confianza interpersonal que los hombres. 


\section{LA CONFIANZA EN LAS INSTITUCIONES}

Una tarea fundamental de la formación ciudadana es conseguir la confianza en los otros que no se conocen, simplemente porque se confía en las instituciones y las reglas de la democracia. De aquí que la confianza en las instituciones pertenece a la esfera pública y se construye en base a fuentes secundarias, en especial a los medios de comunicación de masas. La confianza en las instituciones, sostienen Montero, Zmerli y Newton (2008:21), «conlleva la creencia de que ésta no actuará de una forma arbitraria o discriminatoria que resulte dañina para nuestros intereses o los del país, sino que nos tratará, a nosotros y a los demás ciudadanos, de una forma igualitaria, justa y correcta». De esta forma, la confianza en las instituciones se convierte en un importante indicador del sentimiento de los ciudadanos sobre su sistema político.

Los estudios sobre confianza en las instituciones indican que los ciudadanos son capaces de distinguir entre distintos tipos de instituciones con las que se relacionan a la hora de depositar su confianza; es decir, no confían en todas las instituciones de igual forma. A juicio de Rothstein y Stolle (2002), es posible identificar tres tipos distintos de instituciones: las instituciones políticas o representativas (gobierno, parlamento y partidos políticos), las instituciones imparciales o de orden (policía, ejército, justicia, escuelas, sistema de salud), y las instituciones de control (prensa, funcionarios o empleados públicos), a la que podríamos también agregar, en este último caso, a la iglesia, que en el caso de América Latina, tiene aún una importante cuota de control.

Si se observa el nivel de confianza de cada institución por país —señalan los datos del Latinobarómetro-vemos enormes diferencias. En Chile, por ejemplo, la confianza en los partidos políticos es de un $23 \%$, muy por debajo del $45 \%$ que se presenta en Uruguay. El estudio realizado por FLACSO/IPSOS (2010:8) sobre Gobernabilidad y Convivencia Democrática en América Latina, arrojó resultados coincidentes a los del Latinobarómetro. «Los latinoamericanos/as depositan una excesiva confianza en los medios de comunicación (noticieros de televisión, de radio, y periódicos) que gozan de un nivel de confianza del $59 \%$, esto es, aparecen con un 24\% más de confianza que las instituciones relacionadas con el Estado (políticos, fuerzas armadas, presidente de la república y presidente del congreso) que alcanza sólo el $34 \%$ de las opiniones favorables».

$\mathrm{Al}$ acercarnos a la situación de la juventud chilena en estas materias, un resultado interesante son los datos que entrega el Informe de 
Chile del Estudio «Educación cívica y el ejercicio de la ciudadanía: los estudiantes chilenos en el estudio internacional de educación cívica» (Mineduc, 2001). Al mirar los porcentajes de confianza hacia las distintas instituciones que se mencionaron a los jóvenes, indica el texto del Mineduc (2001:56), vemos «una gran variación según la institución de que se trate. Mientras que el $51 \%$ de los estudiantes chilenos confía $l a$ mayoría de las veces o siempre en 'la policía', sólo un 11,6\% confía la mayoría de las veces o siempre en 'los partidos políticos'».

El tema de confianza en las instituciones sí es una materia comúnmente presente en las Encuestas Nacionales de Juventud en Chile, pero lamentablemente no es fácil de comparar en las sucesivas mediciones, porque la pregunta y las alternativas de respuestas han variado en el tiempo. No obstante ello, es posible visualizar claras tendencias como se puede apreciar en el resumen que se presenta a continuación.

\begin{tabular}{|c|}
\hline Resultados I Encuesta Nacional de Juventud 1994 \\
\hline $\begin{array}{l}\text { Las instituciones que generan mayor confianza son (respuesta «mucha confianza») } \\
\text { la iglesia }(38,8 \%) \text {; carabineros }(28,8 \%) \text { y radios }(21,4 \%) \text {. } \\
\text { Las instituciones que generan menos confianza (respuesta «nada de confianza») } \\
\text { son, en orden decreciente, partidos políticos }(69,1 \%) \text {, senadores y diputados } \\
(58,2 \%) \text { y sindicatos }(53,7 \%) \text {. }\end{array}$ \\
\hline Resultados II Encuesta Nacional de Juventud 1997 \\
\hline $\begin{array}{l}\text { Las instituciones que concitan el mayor grado de confianza entre los jóvenes son la } \\
\text { iglesia (84\%) y los medios de comunicación masivos (83\%). } \\
\text { Los parlamentarios alcanzan el 32\% de confianza y los partidos políticos el 27\%. }\end{array}$ \\
\hline Resultados III Encuesta Nacional de Juventud 2000 \\
\hline $\begin{array}{l}\text { Las instituciones o instancias más confiables para la mayor cantidad de jóvenes } \\
\text { son los profesores }(51,8 \% \text { y la iglesia católica }(48,1 \%) \text {. } \\
\text { Los que se mencionan como menos confiables son los partidos políticos }(2,7 \%) \text { y } \\
\text { senadores y diputados }(3,0 \%) \text {. }\end{array}$ \\
\hline Resultados IV Encuesta Nacional de Juventud 2003 \\
\hline $\begin{array}{l}\text { El 96,9\% de los jóvenes indica confiar en la familia, le sigue a ello el 81,6\% en las } \\
\text { universidades y el 80,5\% en las escuelas o liceos. } \\
\text { En el otro extremo, sólo un 8,9\% de los encuestados indica confiar en los partidos } \\
\text { políticos y un } 18,4 \% \text { en el congreso, un } 20,3 \% \text { en el poder judicial. }\end{array}$ \\
\hline Resultados V Encuesta Nacional de Juventud 2006 \\
\hline $\begin{array}{l}\text { Al consultar por los grados de confianza en la familia resultó ser, al sumar los } \\
\text { porcentajes de respuesta de confianza total y bastante confianza, la más confiable } \\
(93,1 \%) \text {, seguida de las universidades }(62,4 \%) \text { y la escuela o liceo }(51,8 \%) \text {. } \\
\text { Existe un bajo grado de confianza respecto al gobierno }(17,7 \%) \text {, los tribunales de } \\
\text { justicia }(16,8 \%) \text {, la municipalidad }(16,8 \%) \text {, el poder judicial }(14,2) \text {, el congreso } \\
(9,6 \%) \text { y los partidos políticos }(7 \%) \text {. }\end{array}$ \\
\hline
\end{tabular}


Es claro que a través del tiempo se presenta en forma estable una muy baja confianza en las instituciones vinculadas a instancias políticas y judiciales, siendo los partidos políticos los que menos confianza suscitan en los jóvenes chilenos.

Estos datos reflejan además que instituciones como la iglesia y los medios de comunicación, ambos muy importantes en la formación de la opinión, van pasando a una posición intermedia, ya no quedan en los de mayor confianza, pero tampoco ingresan entre los que reúnen baja confianza.

Por otro lado, es importante notar cómo las escuelas y las universidades se empiezan a instalar en los primeros lugares de las instituciones públicas confiables, lo que habla de un interés de los jóvenes por integrarse a la sociedad a través de estos servicios — que en el caso de Chile tienen bastante de privado y cada vez menos de público- - , que son en definitiva medios a través de los cuales canalizan sus esfuerzos personales.

En la última medición, la VI Encuesta Nacional de Juventud (2009), se reitera lo anterior, los centros de formación presentan la más alta confianza y los partidos políticos la más baja. La iglesia católica y los medios de comunicación se ubican en un punto medio, en especial estos últimos, que tienen un nivel de baja confianza muy similar al nivel de alta confianza.

\begin{tabular}{|c|c|c|}
\hline $\begin{array}{c}\text { Baja confianza } \\
\text { Notas 1 a 3, Porcentajes }\end{array}$ & $\begin{array}{c}\text { Alta Confianza } \\
\text { Notas 8 a 10, Porcentajes }\end{array}$ \\
\hline $7,2 \%$ & Universidades & $38,8 \%$ \\
\hline $8,7 \%$ & Escuelas/liceo & $31,0 \%$ \\
\hline $18,8 \%$ & Carabineros & $27,4 \%$ \\
\hline $34,0 \%$ & Iglesia católica & $24,6 \%$ \\
\hline $18,9 \%$ & Medios de comunicación & $21,1 \%$ \\
\hline $35,4 \%$ & Poder judicial & $10,3 \%$ \\
\hline $28,9 \%$ & Municipalidad & $9,9 \%$ \\
\hline $48,3 \%$ & Congreso & $2,5 \%$ \\
\hline $59,0 \%$ & Partidos políticos & $2,2 \%$ \\
\hline
\end{tabular}

Fuente: VI Encuesta Nacional de Juventud, Chile, 2009.

Al analizar lo anterior con más detalles, se puede apreciar que la confianza varía según edad. Los jóvenes de menor edad son los que señalan confiar más, en general, en las instituciones y sólo en la municipalidad se rompe esta tendencia. A su vez, los jóvenes de sectores rurales tienen mayor confianza en las instituciones que quienes viven en la ciudad. 
La VI Encuesta Nacional de Juventud profundiza también en el tema consultado por confianza en personas y/o cargos. Situación que posibilita diferenciar de mejor forma entre instituciones públicas y personas con las que se comparte cotidianamente en la vida privada. Los jóvenes tiene mayor confianza en personas asociadas al entorno íntimo e inmediato, más que en actores institucionales, y más aún si estos últimos se asocian al mundo político. La alta confianza en la familia es de un $81,5 \%$ mientras que la «alta confianza» en senadores y diputados es de sólo un 2,7\%.

El valor que los jóvenes asignan a la familia y a las amistades puede estar reflejando una privatización de la vida social, de manera que ésta aparece como fuente de apoyo y probablemente fuente de sentido para los jóvenes en una sociedad de mayor inestabilidad.

Los datos de esta última encuesta también demuestran que hay coherencia entre los niveles de confianza en las instituciones y en las personas asociadas a ellas. A modo general, existe coincidencia en el nivel de confianza hacia las instituciones y las personas que pertenecen a ellas. Sin embargo, sí se produce una brecha mayor en dos de las instituciones que han venido bajando en niveles de confianza: la iglesia y los medios de comunicación social. En ambos casos, la confianza en la institución es mayor que en las personas. Esto podría estar indicando un cierto desencanto de personas en particular, lo que es posible en el caso de la iglesia en los últimos años, por acusaciones en particular a religiosos.

\section{ALGUNAS CONSIDERACIONES PARA COMPRENDER LA BAJA CONFIANZA SOCIAL DE LA JUVENTUD}

Dos consideraciones previas, una que resulta obvia de la presentación de los datos. No es que la juventud chilena sea desconfiada en particular, sino que en general la población latinoamericana, en gran medida, no presenta altos niveles de confianza en los otros.

Lo anterior se puede apreciar en datos comparativos entre regiones, lo que puede verse en el informe Estudio Mundial de Valores (World Values Survey), donde en Europa evidentemente los niveles de confianza en los otros no conocidos son mucho mayores que en Latinoamérica. ${ }^{1}$

1 A modo de ejemplo, en el World Values Survey (Wvs) 1995-1997, el nivel agregado de confianza social o interpersonal (definido como el porcentaje de encuestados diciendo que «se puede confiar en la mayor parte de la gente») va de un 65,3\% en Noruega, hasta un 2,8\% en Bra- 
Estudios como el de la Fundación BBVA (2006), ubican también a Chile entre los países de menor nivel de confianza en los otros. De trece países encuestados, once de ellos, en una escala de 0 a 10 , se ubican sobre 5, mientras que Chile y Turquía están bajo 5. Este estudio, además, da cuenta de que Chile es también un país donde su población confía menos en los jóvenes que en los adultos. La población chilena responde a la pregunta «en quién se puede confiar más», indicando en los adultos el $66,2 \%$; en los jóvenes 7,4\%; indistintamente en jóvenes o adultos $25,4 \%$ y no responde $1,0 \%$. En países como España las cifras respectivamente son: $30,2 \% ; 13,1 \%$; $53,7 \%$ y $3,0 \%$.

Otro aspecto importante dentro de esta primera consideración, es que la realidad de Chile no es muy lejana al resto de los países de América Latina. A este respecto es posible encontrar numerosos artículos sobre la materia que dan cuenta de situaciones similares de baja confianza social en general en los diferentes países latinoamericanos como Argentina (Jorge, 2006), Brasil (Power, 2002) o Venezuela (Reverón y Vargas, 2009).

Una segunda consideración es algo más técnica, principalmente en lo que respecta a confianza en los otros. A juicio de Yáñez, Ahumada y Cova (2006:14-15), resumiendo a varios investigadores que han escrito sobre la materia, cuestionan la forma tradicional de preguntar para medir confianza en los otros. Las críticas a esta forma de medición de la confianza social son varias, se cuestiona que la pregunta no ofrezca elecciones intermedias entre los dos extremos (dos alternativas: acuerdo-desacuerdo) y que no esté contextualizada; se critica también que la pregunta en cuestión no pida a las personas responder entre confiar y desconfiar, sino entre confiar y ser precavido. Para estos autores, las personas pueden confiar en los otros y a la vez creer que es prudente ser precavido.

Yañez, Ahumada y Cova (2006) cuestionan que la desconfianza sea la ausencia de confianza y plantean que ambas actitudes son independientes, lo que permitiría distinguir entre la actitud ingenua y la actitud prudente en la confianza. Para estos autores, al abordar la confianza social se debe superar la perspectiva normativa que ve la confianza como positiva y la desconfianza como negativa, y por ello hacen la distinción entre ingenuos y prudentes en la construcción de confianza en las relaciones interpersonales. A juicio de estos autores,

sil. En el caso de Chile, se ubica en 21,4\%. Ningún país latinoamericano supera el $30 \%$. 
en la actualidad, y más aún en una sociedad de mayor complejidad y transitoriedad en las relaciones interpersonales (citando a Bauman), pueden coexistir simultáneamente tendencias a confiar y tendencias a desconfiar en las personas.

Otro cuestionamiento técnico a la forma de medir confianza cuantitativamente es que el indicador ocupado habitualmente para medir confianza en los otros parece que no es adecuado para la región, ya que la confianza interpersonal abierta a terceros desconocidos no es un fenómeno habitual en nuestras sociedades latinoamericanas. En Chile y en la región latinoamericana, la confianza se manifiesta frente a personas que se conocen, con las cuales se tiene por experiencia de vida algún tipo de intercambio. La confianza se desarrolla en medio de la familia, los amigos, las personas con las que se trabaja, en síntesis, con las personas con que se interactúa.

Para superar este cuestionamiento, sería necesario hacer la distinción entre asociatividad y sociabilidad introducida por Valenzuela y Cousiño (2000:321). A juicio de estos autores, si bien «la disposición a confiar en los demás está en el origen de la aptitud típicamente norteamericana para establecer relaciones sociales con desconocidos, que se expresa en la fortaleza de sus relaciones de vecindad, amistad y asociatividad. La sociedad chilena, en cambio, se caracteriza por tener umbrales de confianza social muy bajos, que resienten toda la estructura de relación con extraños. La debilidad asociativa que resulta de ello se compensa, sin embargo, con la fortaleza y densidad que adquieren las relaciones entre conocidos, cuyo fundamento se encuentra en el espacio de las relaciones familiares y que definen, por contraste con el modelo de la asociatividad norteamericano, el modelo de la sociabilidad que es propio de nuestra sociedad».

No obstante estas dos precisiones, no podemos dejar de reconocer a partir de los datos presentados que hay una baja confianza en los otros considerados en general, como también una baja confianza en las instituciones sociales, en especial las del mundo de la política. Ésta es una realidad que es evidente en Chile desde fines de la década de 90, con trabajos como Las paradojas de la modernización (PNUD, 1998), que responsabilizan a la desconfianza y al temor al otro como frenos a la sociabilidad democrática; en las reflexiones contenidas en el texto editado por el Ministerio Secretaría General del Gobierno (2001:2) titulado "Confianza social en Chile: desafíos y proyecciones», que constata que «el malestar, la falta de confianza en las instituciones y en la personas, una desidia respecto del compromiso social, son esta- 
dos de la cuestión social». Como también en los resultados del PNUD (2002) del informe Desarrollo humano 2002: nosotros los chilenos, un desafío cultural. Informe, este último, que invita a reflexionar la cultura como elemento de un proyecto país, y donde cultura se asume como prácticas de vivir juntos y las representaciones de dicha convivencia. Lechner sostenía (2002) que la falta de confianza debilita el nosotros e inhibe la construcción de lazos.

Mencionadas estas dos consideraciones, es posible ahora profundizar en las interpretaciones que buscan comprender la situación de la confianza social; para ello nos concentraremos primero en lo referido a la confianza en los otros en general y luego, en la confianza en las instituciones.

Hay dos factores principales que han sido señalados tradicionalmente como posibles causas de una baja confianza: una distribución no equitativa del ingreso y la inexistencia de redes densas de asociacionismo cívico. En el primer caso, se visualiza una unidireccionalidad cuando la distribución de la riqueza es regresiva, entonces la confianza tiende a ser baja. En el segundo caso, la relación entre confianza y asociacionismo es más compleja, pues los dos aspectos parecen reforzarse mutuamente.

Si bien la confianza interpersonal, en el marco del Estudio Mundial de Valores, ha buscado probar que hay una relación entre el grado de desarrollo de los países y el nivel de confianza, en Latinoamérica la vinculación es de otro orden. «La paradoja de América Latina -indica el Informe Latinobarómetro (2010:71) — es que se puede crecer y ser desconfiado a la vez. En el caso de esta región, no se confirma la hipótesis de Inglehart —se hace referencia a Ronald Inglehart, quien diseñó el indicador de confianza interpersonal en el Estudio Mundial de Valores- de que el crecimiento económico está asociado a mayores grados de confianza».

Probablemente la explicación a esta paradoja es que la falta de equidad, más que el crecimiento económico, explica la desconfianza en los otros, ya que la riqueza absoluta de una sociedad sería menos importante que el modo como está distribuida. El grado en que el bienestar está extendido parece que determina si es racional confiar o no en los demás. En este ámbito, como sostiene la CEPAL (2007) en su trabajo «Cohesión social: inclusión y sentido de pertenencia en América Latina y el Caribe», el desarrollo latinoamericano muestra un casillero vacío, pues no logra conjugar crecimiento y equidad. 
No es que no exista confianza en nuestras sociedades, señala el Informe Latinobarómetro (2010:71), es sólo que existe entre quienes se conocen: las familias y los amigos. «Nuestras sociedades están relativamente parcializadas por esas redes de confianza, nuestras ciudades no tienen integración de ricos y pobres en el mismo lugar, sino más bien existen los barrios de los ricos y los barrios de los pobres que no interactúan para nada entre sí. Eso mismo sucede con los empresarios, los militares, los políticos, etcétera. Cada cual tiene su propio ámbito de funcionamiento donde la interacción con los otros grupos de la sociedad es poca y con pocos».

Esta fragmentación coloca en una actitud de desconfianza frente al desconocido, a lo que se agrega que la vulnerabilidad y la incertidumbre son hoy parte de la vida diaria a causa de la imprevisibilidad e inestabilidad que plantea el nuevo capitalismo, que en el caso de América Latina se ha llevado a niveles extremo, siendo Chile un claro ejemplo de ello. Las transformaciones adoptadas en la actualidad en el mundo del trabajo (flexibilidad, cortoplacismo, inmediatez) han generado una significativa modificación en el carácter de las personas. Sennett (2000) indica que «nada a largo plazo», es el principio que corroe la confianza, la lealtad y el compromiso mutuo.

A juicio de Bauman (2008:9) «podemos afirmar que el tipo de inseguridad actual se distingue claramente por el temor al crimen y a los malhechores. Predomina la desconfianza en los demás y en sus intenciones, así como también una actitud que niega o considera imposible tener fe en la constancia y en la fiabilidad del compañerismo humano. Castel, ${ }^{2}$ atribuye esta situación al individualismo contemporáneo; sugiere que la sociedad de hoy, al suprimir las comunidades y corporaciones estrechamente unidas que antes las leyes delimitaban y velaban por su cumplimiento y sustituirlas por el deber de ocuparse cada uno de sí mismo y de sus asuntos, se ha edificado sobre el terreno pantanoso de la incertidumbre».

Esta realidad de nuestras sociedades tiene impacto también —afirma el Informe Latinobarómetro (2010: 71) — «sobre las actitudes de la población hacia las instituciones, ya que por definición están fuera del ámbito de las relaciones directas de cada cual. Confiar en algo que no se conoce no está de acuerdo con esta estructura social parcializada que tienen nuestras sociedades».

2 Bauman está haciendo referencia al trabajo de Robert Castel (2003): L'insécurité sociale: Qu'est-ce qu'être protégé? París: Editions du Seuil. 
No obstante lo anterior, no resulta adecuado pensar en la confianza como un rasgo cultural inmutable de la sociedad, sino más bien como una reacción relativamente contingente que se relaciona con la percepción acerca de la capacidad de las instituciones de sintonizar con las demandas ciudadanas, realizando esfuerzos —en términos de política pública - para gestionar soluciones a dicha demanda. En América Latina, en este aspecto, parece primar una racionalidad utilitaria más que axiológica a la hora de evaluar las instituciones. Es decir, los ciudadanos confiarán más en aquellas instituciones que sean percibidas como eficaces a la hora de cumplir con sus funciones.

Por lo tanto, la confianza hacia las instituciones no es una expresión de la simple afectividad positiva o negativa hacia una institución particular, sino que es un juicio que resume diversos aspectos de la institución evaluada; y por tanto, incluye y combina diversos componentes evaluativos - algunos más estables y centrales, otros más contingentes y periféricos-. En particular, sostiene Segovia et al. (2008:40) hay dos componentes fundamentales «las percepciones de los ciudadanos respecto de la capacidad de una institución para realizar las acciones que se espera de ella, y las percepciones respecto de la voluntad o interés de la misma para actuar en pos del bienestar de la sociedad». En síntesis, las personas atribuyen confianza a una institución, principalmente, a partir de considerar que dicha institución puede hacer lo que de ella se pide en virtud de sus competencias, y que efectivamente quiere hacerlo.

No obstante todo este marco de fragmentación y desconfianza del otro, más allá de las particularidades nacionales, indica la CEPAL/OIJ (2008:307) en Juventud y cohesión social en Iberoamérica: un modelo para armar: «cabe preguntarse cómo entender el optimismo de los jóvenes latinoamericanos; es decir, las expectativas compartidas de movilidad social tanto en el mediano como en el largo plazo, si es que una amplia mayoría de ellos piensa que no hay igualdad de oportunidades. Una posible respuesta es que hoy transitamos desde una posición de crítica sistémica, que responde a una postura ideológica o a un desencanto vital, hacia una validación del emprendimiento, que bien puede ser crítica en el nivel general sin renunciar a la confianza en los esfuerzos individuales propios. En cierta forma, esto implicaría el tránsito hacia una visión más liberal de la estructura social».

En este marco general de desconfianza y de una juventud que valida el emprendimiento, se está dando vida a una cohesión social ya no construida sobre la homogeneidad, sino sobre la base de que a nadie se 
le discrimina y donde el esfuerzo individual no descarta el apoyo público, sino al contrario, lo demanda como una compensación al trabajo individual.

Ser joven, en esta realidad, resulta muy difícil, pues cada uno tiene que construirse con sus propias fuerzas un sentido de futuro, definir un espacio y lograr un reconocimiento de los demás. De aquí que en las últimas Encuestas Nacionales de Juventud en Chile es muy notorio el peso que tiene la iniciativa individual, el esfuerzo personal y la responsabilidad en el logro de las aspiraciones.

SANTIAGO (CHILE), ENERO 2011

RECIBIDO: FEBRERO 2011

ACEPTADO: MARZO 2011

\section{REFERENCIAS BIBLIOGRÁFICAS}

BAEZA, JoRge y MARIO SANDOVAL (2009): «Valores en estudiantes de educación media de la Región Metropolitana». Santiago: CEJU/UCSH (inédito). (2007): «Configuración de valores en jóvenes estudiantes secundarios de la Región Metropolitana». Boletín de Investigación Educacional, 22 (2). Santiago: PUC.

BAuman, Zygmunt (2008): Confianza y temor en la ciudad. Barcelona: Arcadia.

BBVA (2006): «Estudio internacional sobre capital social». Disponible en: www.grupobbva.com.

CEPAL (2007): Cohesión social: inclusión y sentido de pertenencia en América Latina y el Caribe. Santiago: CEPAL.

— y OIJ (2008): Juventud y cohesión social en iberoamérica: un modelo para armar. Santiago: CEPAL/OIJ.

CiEPLAN/IFHC (2007): «Encuesta de cohesión social en América Latina (ECosociaAL)». Disponible en: www.ecosocialsurvey.org.

FLACSO/IPSOS (2010): «Estudio de opinión pública en Latinoamérica 20092010: gobernabilidad y convivencia democrática en América Latina». San José: FLACSO.

HerReros VÁzquez, Francisco (2004): «¿Por qué confiar? Formas de creación de confianza social». Revista Mexicana de Sociología, 66 (4). México: UNAM.

HunNeuS, CRISTÓBAL (2010): «Una nueva mirada al mundo del trabajo. Seminario políticas laborales». Disponible en: www.fes.cl.

INJUV (2009): Sexta encuesta nacional de juventud. Santiago: INJUV. 
(2006): Quinta encuesta nacional de juventud. Santiago: INJUV.

(2003): Cuarta encuesta nacional de juventud. Santiago: INJUV.

(2000): Tercera encuesta nacional de juventud. Santiago: INJUV.

- (1997): Segunda encuesta nacional de juventud. Santiago: INJUV.

(1994): Primera encuesta nacional de juventud. Santiago: INJUV.

JoRGE, JosÉ EDUARDO (2006): «La confianza interpersonal en la Argentina». Revista Question. Disponible en: www.perio.unlp.edu.ar.

LAPOP (2010): «Cultura política de la democracia, 2010. Consolidación democrática en las Américas en tiempos difíciles: informe sobre las Américas». Disponible en: www.vanderbilt.edu.

LATINOBARÓMETRO (2010): «Informe 2010».

Disponible en: www.latinobarómetro.org.

LECHNER, NORBERT (2002): «Los desafíos políticos del cambio cultural». Nueva Sociedad $\mathrm{N}^{\circ} 184$. Caracas: Nueva Sociedad.

MinEDUC (2001): «Informe de Chile del estudio educación cívica y el ejercicio de la ciudadanía: los estudiantes chilenos en el estudio internacional de educación cívica». Santiago: Mineduc.

MsgG (2001): Confianza social en Chile. Desafíos y proyecciones. Santiago: MSGG.

MONTERO, JosÉ RAmón; SONJA ZMeRLi y Ken NeWTON (2008): «Confianza social, confianza política y satisfacción con la democracia». REIS, Revista Española de Investigaciones Sociológicas $\mathrm{N}^{\circ} 122$. Madrid: CIS.

PNuD (2002): Desarrollo humano en Chile: nosotros los chilenos un desafío cultural. Santiago: INJUV.

- (1998): Desarrollo humano en Chile: las paradojas de la modernización. Santiago: PNUD.

Power, TiмотнY J. (2002): «La confianza interpersonal brasileña en perspectiva comparada». América Latina Hoy N³2. Salamanca: Ediciones Universidad de Salamanca.

REVERÓn Escobar, ZAira y Adolfo VARgas CACiQue (2009): «Venezuela: confianza V/s desconfianza en las instituciones y gobernabilidad». Cuestiones Políticas, 25 (42). Zulia: Universidad de Zulia.

Rothstein, Bo y DietLind STOLLe, (2002): «How Political Institutions Create and Destroy Social Capital: An Institutional Theory of Generalized Trust». In $98^{\text {th }}$ Meeting of the American Political Science Association, Boston.

Segovia, C.; A. Haye, R. GonzÁlez, J. Manzi y H. CaRvacho (2008): «Confianza en instituciones políticas en Chile: un modelo de los componentes centrales de juicios de confianza». Revista de Ciencia Política, 28 (2). Santiago: PUC.

SENNETT, RICHARD (2000): La corrosión del carácter. Barcelona: Anagrama. Simmel, GEORG (1906): «The Sociology of Secrecy and of Secret Societies» Disponible en: www.brocku.ca. 
USlAnER, ERIC (2002): The Moral Foundations of Trust. Cambridge: Cambridge University Press.

VALENZUELA, EDUARDO y CARLOS COUSIÑO (2000) «Sociabilidad y asociatividad: un ensayo de sociología comparada». Estudios Públicos Nº77. Santiago: Centro de Estudios Públicos.

YÁÑEZ, AHUMADA y Cova (2006): "Confianza y desconfianza: dos factores necesarios para el desarrollo de la confianza social». Universitas Psicológica, 5 (1). Bogotá: Pontificia Universidad Javeriana. 